
\title{
25 Research Soure \\ The time course of acute Percheron artery ischemic coma on imaging: a retrospective cohort study
}

\section{Dao-Ming Tong ( $\nabla$ dmtong@xzhmu.edu.cn )}

Affilicated Shuyang People' Hospital,Xuzhou Medical University https://orcid.org/0000-0002-45672439

\section{Ye-Ting Zhou}

Affiliated Shuyang Hospital

\section{Guo-Hong Liu}

Affiliated Shuyang Hospital

\section{Research article}

Keywords: Stroke, Aetery of Percheron infarction, time course, coma, incidence, awakening events, mortality

Posted Date: December 30th, 2019

DOl: https://doi.org/10.21203/rs.2.19706/v1

License: (c) (1) This work is licensed under a Creative Commons Attribution 4.0 International License. Read Full License

Version of Record: A version of this preprint was published at Brain Research Bulletin on December 1st, 2020. See the published version at https://doi.org/10.1016/j.brainresbull.2020.10.016. 


\section{Abstract}

\section{Background}

We investigated the time course of lesions for awakening in acute Percheron artery ischemic coma (PAIC), which was previously unknown.

\section{Methods}

Patients who had newly identified acute PAIC events in 2011-2014 and had not received thrombolytic therapy were enrolled retrospectively. The time course of lesions in PAIC was investigated by diffusionweighted imaging (DWI).

Results

Ninety-three patients met the inclusion criteria, of whom 63 and 30 had transient PAIC and persistent PAIC, respectively. The time course of awakening events in persistent PAIC decreased over time, with large lesions in the bilateral paramedian thalamus/ rostral midbrain on DWI almost in all patients who was either plus or minus a "top of basilar artery" strokes. Whereas awakening events in transient PAIC increased over time, with small or lacunar lesions in the unilateral or bilateral thalamus/rostral midbrain about in $30.2 \%$ cases, and the rest in naturally recanalization of infarcts or TIA. Lesion volumes were larger for persistent PAIC than for transient PAIC (median, $2.4 \mathrm{~cm} 3$ vs. $0.03 \mathrm{~cm} 3, \mathrm{P}<0.0001$ ). In Cox hazards ratio (HR) analysis, a lower GCS score was associated with mortality (HR, 5.5; $95 \%$ confidence interval [Cl],1.427-21.45). Multivaliate analysis shown that the predictors of higher risk of persistent PAIC were only increased NIHSS scores (HR,1.3; 95\% Cl,1.109-1.640) and large lesions in bilateral thalamus/rostral midbrain (HR,15.0; $95 \% \mathrm{Cl}, 1.440-58.13)$.

\section{Conclusions}

The only two forms of acute PAIC events were transient and persistent. Most persistent PAIC was associated with large lesions in bilateral paramedian thalamus/rostral midbrain, and with high mortality.

\section{Background}

Acute coma is a clinical emergency[1, 2]. Acute Percheron artery ischemic coma (PAIC) refers to acute coma caused by ischemic event in the Percheron artery region[3-7]. In recent years, there are more frequent trends in the reports on acute PAIC events[5-10]. Moreover, the imaging spectrum of Percheron artery infarction have been reported[11]. Importantly, severe PAIC represent life-threatening emergencies, and their prognosis is very poor[11-13]. However, the time course of acute PAIC events on magnetic resonance imaging (MRI) is still unknown. In the present study, we investigated this time course on MRI for acute PAIC events through a 3-year follow-up period. Our hypothesis was that the large lesion in the paramedian thalamus/rostral midbrain bilaterally would result in the persistent PAIC and increased mortality, whereas the small or lacunar lesion in the paramedian thalamus/rostral midbrain would result 
in the transient PAIC and had a good outcome. The aims of our study was to determine whether the time course of acute PAIC on MRI was associated with a different lesions for awakening events.

\section{Methods}

\section{Study settings}

We used the integrated medical records system of a tertiary teaching hospital to retrospectively analyze patients admitted to the neurology ward or the intensive care unit (ICU) stay because of acute PAIC events.

\section{Related definitions}

\section{Patients and selection criteria}

The current study patients were hospitalized because of a diagnosis of acutely coma with an emergency brain computed tomography (CT) and MRI, and were subsequently discharged between July 1, 2011 and July 1, 2014. Of these patients, 93 were identified as having developed acute PAIC events on MRIdiffusion- weighted images (MRI-DWI) within 48 hours of symptom onset. All patients were admitted within 24 hours and underwent an emergency brain CT scan that excluded intracranial hemorrhage. All patients received non-thrombolytic therapy during the duration of hospitalization because this hospital did not rise to the grade using thrombolytic treatment at that time. The acute PAIC were assessed using the Glasgow Coma Scale $(\mathrm{GCS})$ motor score $(\mathrm{GCSM})<6$. The lesions of PAIC events were investigated using MRI-DWI.

The diagnostic criteria of acute PAIC events were as follows: (1) an acute coma of a GCS total score $\leq 8$ and a GCSM score $<6$ were required; (2) for diagnosis of the PAIC had to have been based on either a positive lesion or suspected a negative ischemic event on DWI, which was located between the unilateral/bilateral paramedian thalamus/rostral midbrain.

The exclusion criteria were as follows: (1) a GCS total score $\geq 8$ or a GCSM score $=6$; (2) a cause of coma caused by bilateral diffuse cerebral cortex lesions or secondary thalamic injure due to compression from supratentorial herniation; (3) a primary injure located between caudal midbrain-pontine; and (4) non-PAIC or other conditions of impaired consciousness (see the supplementary Table I).

Related Definitions

Coma is a state of unconsciousness from which the patient cannot be aroused, including the absence of language and motor function $(\mathrm{GCSM}<6)$. Stupor refers to a state of unconsciousness from which patient can be roused only by strong stimulation. Awakening is defined as the restoration of command following $(\mathrm{GCSM}=6)$.

The location of the neural network for arousal is limited between the thalamus and the upper brainstem bilaterally[14], and the blood supply in the bilateral paramedian thalamus/rostral midbrain comes from the thalamic penetrating artery (TPA), which is arising from both P1 segment of the posterior cerebral artery (PCA).(Fig. 1A) So-called Percheron artery refers to a variant TPA arising from the proximal of the unilateral PCA and then supply to the paramedian thalamus and the rostral midbrain bilaterally[11], 
(Fig. 1B) which is presented with one third of the population[3].The PAIC relationships with events are provided in the supplementary Table II.

In this study, transient PAIC refers to a PAIC of less than $24 \mathrm{~h}$ in duration; and persistent PAIC refers to a PAIC that last more than $24 \mathrm{~h}$. We selected patients with the paramedian thalamus/ rostral midbrain ischemic events with DWI lesion $\leq 3 \mathrm{~mm}$ in axial diameter as "small" lesion, DWI lesion $>3 \mathrm{~mm}<15 \mathrm{~mm}$ as "lacunar" lesion, and DWI lesion > $15 \mathrm{~mm}$ in axial diameter as "large" lesion.

Data extraction

MRI was performed with 1.5-T equipment (Siemens AG, Munich, Germany). MRI sequences included DWI, fluid-attenuated inversion recovery (FLAIR), and conventional T2 and T1-weighted images, from medulla to cortex, at 5-mm section thickness. The MRI findings in patients with PAIC were verified by a neuroimaging expert and a highly qualified neurologist. MRI infarct volume (in $\mathrm{cm}^{3}$ ) was calculated as the sum of the paramedian thalamus/rostral midbrain infarct area in each MRI slice according to the formula $\mathrm{A} \times \mathrm{B} \times \mathrm{C} / 2$. For irregular shapes, MRI infarct volume (in $\mathrm{cm}^{3}$ ) was directly calculated in the hand painting automatically generated area $\left[\mathrm{mm}^{2} \times\right.$ (slice thickness $5 \mathrm{~mm} \square$ interlayer spacing $1 \mathrm{~mm}$ )] on the picture archiving conmunication system (PACS).

All data were extracted from medical records. Data were collected on the patient's age, gender, home address, admission date, ICU stay, and duration of hospital stay. The GCS scores were recorded based on descriptions of neurological examination findings at admission. The National Institutes of Health Stroke Scale (NIHSS) scores and ABCD2 scores were calculated based on the patient's medical records on admission.

To investigate the time course of patients in comas, information was obtained from a hospital medical registration database or patients were interviewed by a neurological specialist (TDM). Survival results were initially determined from the hospital database or based on follow-up telephone interviews with the abovementioned neurologist. Prognostic information was evaluated using the mRS (scores range from 0 to 6 , no symptoms $=0$, slight symptoms $=1$, restriction $=2$, slight disability $=3$, moderate disability $=4$, severe disability $=5$, death $=6$ ). If the patient died after discharge, information was obtained from the patient's closest living relative. Follow-up began in January 2012 and ended in Dec 2014.

Statistical analysis

All numeric variables are expressed as mean \pm standard deviation (SD) or median (interquartile range [IQR]). Fisher's exact test and the Mann-Whitney $U$ test were used to examine the relationship between baseline patient variables. Continuous variables were compared using Student's t test. A binary logistic regression analysis was performed for each dichotomous baseline factor. Survival estimates were obtained using the Kaplan-Meier method and the log-rank test was performed to assess the statistical significance of differences in survival. Multivariate regression analyses were performed for each baseline factor using the Cox proportional hazards model. The statistical analysis was conducted in SPSS version 17.0 (SPSS Inc., Chicago, IL, USA). P-values $<0.05$ were considered statistically significant.

\section{Results}

We screened 918 acute comatose patients who were admitted to our neurologic ward during a 3-year period. We excluded 459 patients with hemorrhagic stroke, 234 patients with ischemic stroke in the 
cerebral anterior circulation area, and 132 patients with non-PAIC. Ultimately, only 93 patients with acute PAIC events were met the eligibility/exclusion criteria and included in the analysis.

The mean age was $68.9 \pm 11.9$ years and $45(48.4 \%)$ patients were male. Based on the time course of awakening events among patients with acute PAIC, we diagnosed 63 patients with acute transient PAIC and 30 patients with acute persistent PAIC events.

Among the 93 patients with acute PAIC events, 13 experienced sudden dizziness, 3 transient and 7 persistent PAIC experienced sudden seizure, two experienced sudden numbness of an arm and leg, and one presented suddenly weakness of an arm and leg as precursors, while the remaining 67 patients experienced no warning.

The 30 patients with persistent PAIC events underwent a brain DWI within 24-48 h of admission, which was validated the diagnosis of coma caused by acute ischemic infarction in the Percheron artery territories. Of the 63 patients with transient PAIC events, 60 (95\%) within 24-48 h of admission received a DWI examination (remaining 3 received a single negative head CT scan). The interval from onset to the time of the MRI did not differ significantly between transient PAIC and persistent PAIC ( $29.8 \pm 12.8 \mathrm{~h}$ vs $31 \cdot 4 \pm 16 \cdot 1 \mathrm{~h}, \mathrm{p}=0 \cdot 725)$. On admission, the baseline clinical characteristics in patients with PAIC are showed in the Table 1. 
Table 1

Baseline clinical characteristics in patients with PAIC, $N=93$

\begin{tabular}{|c|c|c|c|}
\hline Variable & $\begin{array}{l}\text { Transient } \\
\text { PAIC }(n=63)\end{array}$ & $\begin{array}{l}\text { Persistent } \\
\text { PAIC }(m=30)\end{array}$ & $\begin{array}{l}\mathrm{P} \\
\text { Value }\end{array}$ \\
\hline 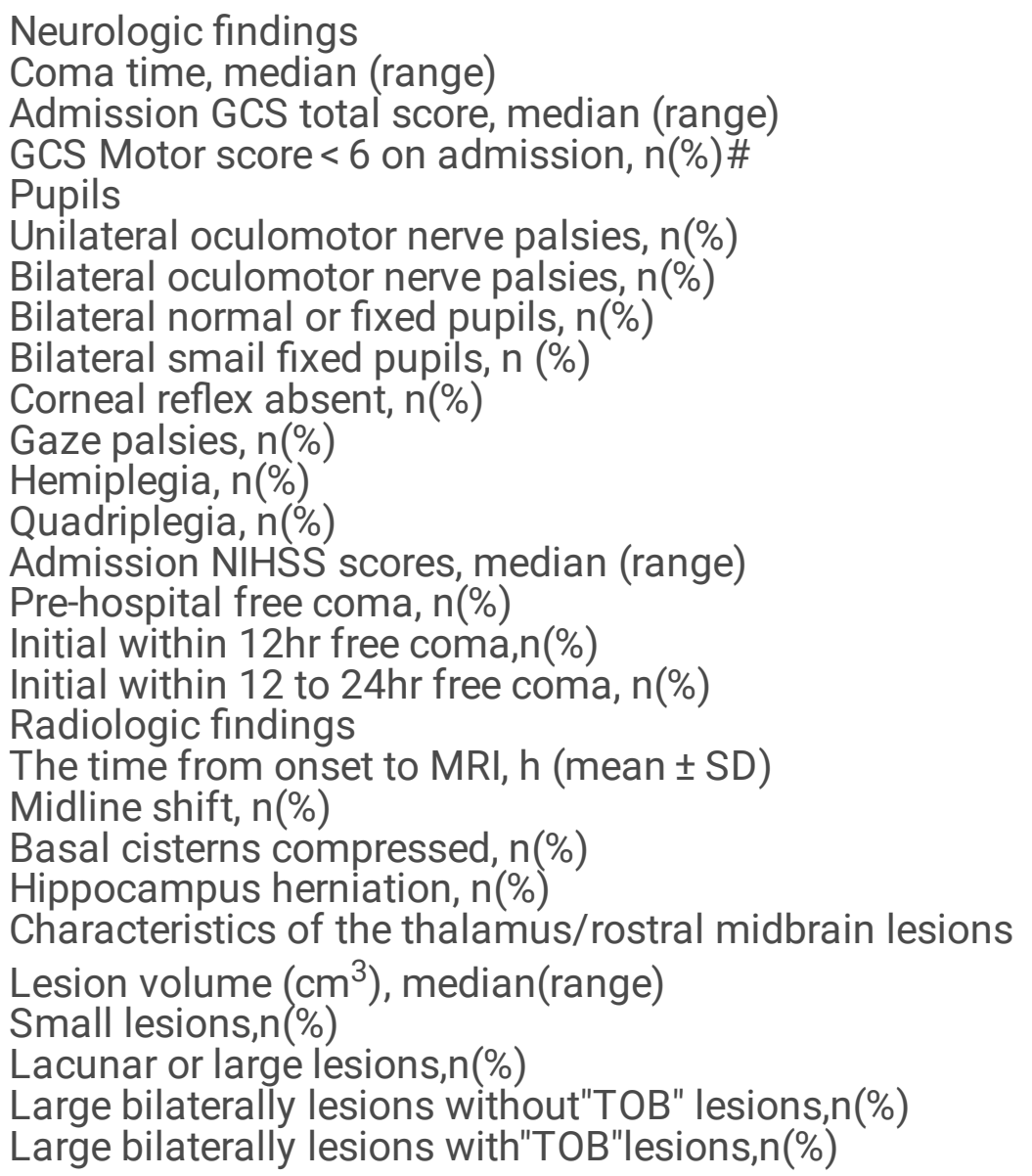 & $\begin{array}{l}1 \mathrm{~h}(0.17-20 \mathrm{~h}) \\
14 \cdot 0(4-15) \\
17(27.0) \\
2(3.2) \\
0(0.0) \\
59(93.7) \\
2(3.2) \\
0(0.0) \\
1(1.6) \\
1(1.6) \\
0(0.0) \\
3.0(0-40) \\
46(73.0) \\
58(92.0) \\
5(0.8) \\
29 \cdot 8 \pm 12 \cdot 8 \\
0(0.0) \\
0(0.0) \\
0(0.0) \\
0.03(0-0 \cdot 8) \\
13(20.6) \\
6(9.5) \\
0(0.0) \\
0(0.0)\end{array}$ & $\begin{array}{l}7.5 \mathrm{~d}(1-718 \mathrm{~d}) \\
6 \cdot 5(4-8) \\
30(100.0) \\
5(16.7) \\
1(3.3) \\
19(63.3) \\
5(16.7) \\
0(0.0) \\
6(20.0) \\
6(20.0) \\
5(16.7) \\
39 \cdot 0(26-42) \\
0(0.0) \\
0(0.0) \\
0(0.0) \\
31 \cdot 4 \pm 16 \cdot 1 \\
1(3.3) \\
1(3.3) \\
1(3.3) \\
2 \cdot 4(0 \cdot 2-6.1) \\
6(20.0) \\
24(80.0) \\
14(46.7) \\
16(53.3)\end{array}$ & $\begin{array}{l}0.000 \\
0.000 \\
0.000 \\
0.034 \\
0.323 \\
0.000 \\
0.034 \\
-.004 \\
0.004 \\
0.003 \\
0.000 \\
0.000 \\
0.000 \\
0.113 \\
0.725 \\
0.323 \\
0.323 \\
0.323 \\
0.000 \\
1.000 \\
0.000 \\
0.000 \\
0.000\end{array}$ \\
\hline $\begin{array}{l}\text { Treatment and outcome } \\
\text { Antiplatelet, } n(\%) \\
\text { Anticoagulation, } \mathrm{n}(\%) \\
\text { Initial intubation ventilation, } \mathrm{n}(\%) \\
\text { Length of stay in ICU (days), median (range) } \\
\text { mRS score, (mean } \pm \text { SD) } \\
\text { Stupor, } \mathrm{n}(\%) \\
\text { Vegetative state, } \mathrm{n}(\%) \\
\text { Minimally conscious state (\%) } \\
\text { Mortality in } 3 \text { years, } \mathrm{n}(\%)\end{array}$ & $\begin{array}{l}57(90.5) \\
6(9.5) \\
3(4.8) \\
0 \cdot 4(0-4) \\
0 \cdot 8 \pm 1 \cdot 6 \\
0(0) \\
0(0) \\
0(0) \\
3(7.7)\end{array}$ & $\begin{array}{l}18(60.0) \\
12(40.0) \\
18(60.0) \\
2 \cdot 7(0-168) \\
4 \cdot 5 \pm 2.3 \\
4(13.3) \\
6(20.0) \\
2(6.6) \\
18(60.0)\end{array}$ & $\begin{array}{l}0.001 \\
0.001 \\
0.000 \\
0.037 \\
0.000 \\
0.009 \\
0.001 \\
0.038 \\
0.000\end{array}$ \\
\hline \multicolumn{4}{|c|}{$\begin{array}{l}\text { \#The range of scores for the motor component of GCS is } 1 \text { to } 6 \text {, with higher scores indicating a higher } \\
\text { level of consiousness. }\end{array}$} \\
\hline
\end{tabular}

\section{Time course of PAIC due to impaired the bilateral paramedian thalamus/rostral midbrain for arousal}


Among 63 cases of transient PAIC events, $19(30.2 \%)$ of the patients with transient PAIC on DWI revealed an unilateral lacunar lesion (Fig. 2A and B) or bilateral smal lesions in the paramedian thalamus/rostral midbrain. The remaining $44(69.8 \%)$ cases with transient PAIC on imaging did not reveal with distinct ischemic lesions (Fig. $2 \mathrm{C}$ and D). The time course of the awakening events in transient PAIC (median coma time: $1 \mathrm{~h}$, range:0.17-20 h) during the first $24 \mathrm{~h}$ increased over time. Of them, $58(92.1 \%)$ naturally awakened during the window of 0 to $8 \mathrm{~h}$ and only five (7.9\%) naturally awakened during the window of 8 to $24 \mathrm{~h}$ after sudden comas.

The time course of the awakening events in persistent PAIC that showed prolonged durations during the three years decreased over time (median coma time:7.5 days, range:1-718 days). For the 2 to 4 days, the awakening events were in 7 cases, 5 to 7 days in 3 cases, 8 to 30 days in 2 cases, and after 30 days in 2 cases. The rest 16 (53.3\%) cases did no awakening.

Among 30 cases of persistent PAIC events, DWI showed bilateral ischemic lesions in the paramedian thalamus/rostral midbrain in all patients, which was exactly measured by PACS imging areas (per slice). (Fig. 3, B and C) Of them, $80.0 \%$ of lesions in patients with persistent PAIC from Percheron artery infarction was usually large and bilaterally (Fig. 4, A and B), only 5 cases were crossed lesions(Fig. 4, C and D). The remaining $20 \%$ of patients was lacunar lesions bilaterally. In the bilateral paramedian thalamus /rostral midbrain lesions, DWI showed that lesions of over half $(16 / 30)$ of the patients with persistent PAIC were involved in the occipital lobe, upper cerebellum, and/or hippocampus (Fig. 5), their blood supply from the top of basilar artery.

The lesions on images measured, the paramedian thalamus/rostral midbrain lesion volume on DWI was significantly larger in patients with persistent PAIC than in patients with transient PAIC (median, $2.4 \mathrm{~cm}^{3}$ vs. $\left.0.03 \mathrm{~cm}^{3}, \mathrm{P}<0.0001\right)$.

The comparison of risk factors in patients with transient PAIC and persistent PAIC are shown in the Table 2 
Table 2

Comparison of risk factors in patients with transient PAIC and persistent PAIC, $\mathrm{N}=93$

\begin{tabular}{|c|c|c|c|}
\hline Variable & $\begin{array}{l}\text { Transient PAIC } \\
(\mathrm{N}=63)\end{array}$ & $\begin{array}{l}\text { Persistent PAIC } \\
(\mathrm{N}=30)\end{array}$ & $\begin{array}{l}P \\
\text { Value }\end{array}$ \\
\hline Male gender, n(\%) & $26(42.3)$ & 19(63.3) & 0.076 \\
\hline Age, y, $($ mean $\pm S D)$ & $68.3 \pm 12.3$ & $70 \cdot 3 \pm 11 \cdot 0$ & $0 \cdot 458$ \\
\hline Hypertension, n (\%) & $35(55.6)$ & $21(70.0)$ & $0 \cdot 840$ \\
\hline Diabetes mellitus, n (\%) & $9(14.3)$ & $8(26.7)$ & $0 \cdot 162$ \\
\hline $\begin{array}{l}\text { Heart disease, } \mathrm{n}(\%) \\
\text { Atrial fibrillation, } \mathrm{n}(\%) \\
\text { Prior history of stroke, } \mathrm{n}(\%)\end{array}$ & $\begin{array}{l}33(52.4) \\
10(15.9) \\
19(30.2)\end{array}$ & $\begin{array}{l}14(46.7) \\
12(40.0) \\
10(33.3)\end{array}$ & $\begin{array}{l}0.661 \\
0.018 \\
0.813\end{array}$ \\
\hline $\begin{array}{l}\text { Cigarette smoking, } \mathrm{n}(\%) \\
\text { Alcohol drinker, } \mathrm{n}(\%) \\
\mathrm{SBP}, \mathrm{mmHg}(\mathrm{mean} \pm \mathrm{SD}) \\
\text { DBP, mmHg (mean } \pm \text { SD) } \\
\text { Dyslipidemia, } \mathrm{n}(\%) \\
\text { Blood glucose, mmol (mean } \pm \text { SD) } \\
\text { Admission GCS total score, median (range) } \\
\text { Admission NIHSS scores, median (range) } \\
\text { Admission ABCD2 scores, median (range) } \\
\text { Large lesions in thalamus/rostral midbrain, } \mathrm{n}(\%)\end{array}$ & $\begin{array}{l}10(15.9) \\
21(33.3) \\
144.5 \pm 26 \cdot 2 \\
84.3 \pm 13.9 \\
38(60.3) \\
7.3 \pm 3.7 \\
14 \cdot 0(4-15) \\
3.0(0-40) \\
4.2(2-7) \\
6(9.5)\end{array}$ & $\begin{array}{l}6(20.0) \\
11(36.7) \\
161.3 \pm 24.8 \\
89 \cdot 9 \pm 14 \cdot 9 \\
15(50.0) \\
7.5 \pm 3.5 \\
6 \cdot 5(4-8) \\
39 \cdot 0(26-42) \\
5.5(3-7) \\
24(80.0)\end{array}$ & $\begin{array}{l}0.770 \\
0.817 \\
0.007 \\
0 \cdot 120 \\
0.377 \\
0.724 \\
0.000 \\
0.000 \\
0.000 \\
0.000\end{array}$ \\
\hline $\begin{array}{l}\text { Abbreviations: PAIC, Percheron artery ischemic } \\
\text { scale; SBP, systolic blood pressure; DBP, diasto } \\
\text { Stroke Scale; ABCD2, age, blood pressure, clini }\end{array}$ & ressure;NIH & ional Institute & $\begin{array}{l}\text { na } \\
\text { ealth }\end{array}$ \\
\hline
\end{tabular}

Univariate analyses indicated that patients with persistent PAIC were more likely to have a higher rate of atrial fibrillation, lower total GCS scores, increased SBP, increased NIHSS scores and ABCD2 scores, and large lesions in bilateral thalamus/rostral midbrain than the patients with transient PAIC (Table 2). However, after adjusting for age and sex, only inceased NIHSS scores (HR,1.3; 95\% Cl,1.109-1.640) and large lesions in bilateral thalamus/rostral midbrain (HR,15.0; 95\% Cl,1.440-58.13) appeared to be associated with persistent PAIC (Table 3 ).

Table 3

the multivariate regression analysis after adjusting for age and sex,

\begin{tabular}{|lcccc|}
\hline Variable & Standard error & HR & $95 \%$ Cl & P \\
& & & & Value \\
\hline $\begin{array}{l}\text { Infarcts volume in thalamus/rostral midbrain } \\
\text { NIHSS scores }\end{array}$ & 1.199 & 15.09 & $1 \cdot 440-58,13$ & $0 \cdot 024$ \\
\hline $\begin{array}{l}\text { HR, hazard ratio; Cl, confidence interval; SD, standard deviation; NIHSS, National Institutes of Health } \\
\text { Stroke Scale. }\end{array}$ & & & \\
\hline
\end{tabular}




\section{Mortality rate}

In the study cohort of 93 patients, a total of 21 patients died within 3 years. In the persistent PAIC group, 5 patients died in the ICU, and 13 died after hospital discharge within 3 years. In transient PAIC gioup, three patients died after hospital discharge ( two died within one month from heart attacks and one died after six months from unknown causes). The survival rate was higher among patients with transient PAIC than among those with persistent PAIC. The respective mortality rates were 3.2\% $(2 / 63)$ and $40.0 \%(12 / 30)$ at 6 months and $4.8 \%(3 / 63)$ and $60.0 \%(18 / 30)$ at 3 years. As compared with the transient PAIC group, the unadjusted hazard ratio (HR) for death in the persistent PAIC group was $13.5(95 \% \mathrm{Cl}, 10.29-29.31 ; \mathrm{P}=$ 0.000) (Fig. 6). In a multivariate Cox proportional hazards analysis, the interaction between a lower GCS scores and the type of PAIC was also significantly associated with survival (HR, 5.5; 95\% Cl, 1.427-21.45; $P=0.013)$.

\section{Discussion}

This is a first study to assess the time caurse of a large series of patients with PAIC diagnosed by MRIDWI during three consecutive years. Although the PAIC that were from the small case series have frequently been reported[3-11, 15-17], our study used a cohort design. Moreover, our cohort study of PAIC found that acute PAIC accounted for $28.4 \%$ of suddenly comas due to acute ischemic strokes and TIA, although previous studies have indicated that acute loss of consciousness accounted for $20 \%$ of embolic strokes[18], and 34\% of TIAs[19]. More importently, we found that the only two forms of acute PAIC events were transient and persistent.

In the present study, 30 (32.3\%) patients with acute persistent coma events showed new infarcts in the paramedian thalamus/ rostral midbrain bilaterally which were identified by DWI, suggesting that these persistent coma events were caused by acute infarcts of Percheron artery (i.e., PAICs) as the Percheron artery -a variant single thalamic penetrating artery is supply to the paramedian thalamus/rostral midbrain bilaterally.

Among of 30 persistent PAIC events, $46.7 \%$ of lesions was isolated in the paramedian thalamus/rostral midbrain bilaterally. However, $53.3 \%$ of persistent PAIC were presented with new lesions in the upper cerebellum, occipital lobe, and/or hippocampus. Though this is met the "top of the basilar" syndrome criteria[17], we considered that these patients had to have an unique Percheron artery. Thus, our results on DWI revealed that the artery of infarction in persistent PAIC is either at the Percheron artery or at the top of basilar artery with unique Percheron artery.

We also found that the transient PAIC were more likely to occur during three consecutive years. Although only $30.2 \%$ of DWI revealed either bilateral small lesions or unilateral lacunar lesion in the paramedian thalamus/rostral midbrain, the remaining patients should be diagnosed the transient comas with naturally recanalization of infarcts or TIA, which is considered that these ischemic events is from the most specific site of impaired consciousness-Percheron artery territory. 
In the present study, $63(67.7 \%)$ patients with acute transient PAIC awoke naturally within $24 \mathrm{~h}$ after the onset of coma. Moreover, our results showed that rates of awakening in patients with transient PAIC during the first 12-h time window were significantly higher than in those in patients with persistent PAIC. These findings suggest two possible interpretations. First, the natural recanalization may be impacted by the hemodynamic and fibrinolytic systems[20], or the undissolved emboli may be pushed into the surrounding tissue by the vascular endothelial cell holes[21]. Second, unilateral naturally recanalization or the formation of collateral circulation[22] may also be the mechanism of awakening.

The rates of awakening decreased over time for patients with persistent PAIC, while mortality rates increased. Regarding Percheron artery ischemic infarction with conservative treatment, previous studies have mostly shown a poor prognosis[11-13,23], although some reports have noted a relatively better prognosis[8,24]. However, the current study indicated that survival was improved among patients with transient PAIC, while the survival of patients with persistent PAIC showed no improvement. The lesion volumes were larger in the persistent PAIC than in the transient PAIC, which was exactly calculated by the PACS. This involves the following two important issues. First, the transient PAIC usually occurs when the patients has suffered an unilateral ischemic lesion or bilateral small ischemic lesions in the paramedian thalamus/ rostral midbrain. Second, the persistent PAIC can occur when the patients has suffered a larger ischemic lesion in the bilateral paramedian thalamus/rostral midbrain. In the persistent PAIC, blood flow did not undergo natural recanalization, there was a poor outcome, and there was a high risk of death.

In the present study, the different risk factors in patients with transient and persistent PAIC suggests that, among patients with persistent PAIC, risk factors were correlated with atrial fibrillation, increased SBP, lower GCS csores, increased NIHSS scores and ABCD2 scores, and large lesion in bilateral paramedian thalamus/rostral midbrain. However, after controlling for age and sex, the multivariate regression analysis confirmed that only increased NIHSS scores and large lesions in bilateral paramedian thalamus/rostral midbrain were the predictors of higher risk of persistent PAIC (versus transient PAIC),

The main strengths of our cohort study determined that the only two forms of acute PAIC were transient and persistent, but persistent PAIC had a marker of images, i.e., the lesions of Percheron territory infarction plus or minus related involvement of other "top of the basilar" ischemic lesions from the occipital lobe, hippocampal cortex, and cerebellum.

However, the current study is subject to several limitations, including those inherent in retrospective studies. First, the artery of Percheron infarction or emboli has demonstrated by the conventional angiography[11,25], but in our series, only a few of patients performed MRA. However, we believe that the diagnosis of acute PAIC is mainly identified by MRI-DWI. Furthermore, even some transient PAIC did not show a positive lesion on DWI, the diagnosis of transist PAIC should be still considered, because previous study has showed that the negative DWI may not also be fully excluded an acute infarction[26, 27]. Second, the Percheron artery infarction and top of the basilar syndrome with onset seizure has been reported[28, 29], its mechanism of onset seizure may be related to the acute ischemic lesions. Although 3 transient and 7 persistent PAIC experienced sudden seizure in our series, these patients did not presented 
with a prior history of epilepsy, so the primary epilepticus should be excluded. In addition, here is a high prevalence zones of cerebrovascular disease[30], some persistent PAIC may be sent to near tertiary teaching hospital; whereas, the transient PAIC might be a neglected coma events and some patients due to quickly resolved did not be sent to hospital, Therefore, the prevalence of acute PAIC might be underestimated in our study population.

\section{Conclusion}

Acute PAIC events are not rare, which is in $28.4 \%$ of all acute ischemic stroke or TIA. The only two forms of acute PAIC were transient and persistent. The time course of lesions for awakening in transient PAIC differed from that in persistent PAIC. which were involved the ischemic lesions volume in the paramedian thalamus/rostral midbrain bilaterally. As persistent PAIC events were associated with a high mortality, at high prevalence countries of cerebrovascular disease need to be prepared adequate intensive care beds and with capacity of intravascular therapy.

\section{Abbreviations}

PAIC:Percheron artery ischemic coma;DWI:Diffusion-weighted imaging;GCS:Glasgow Coma Scale;NIHSS: National Institutes of Health Stroke Scale;ICU:Intensive care unit

\section{Declarations}

\section{Conflict of interests}

All other authors declare no competing interests.

\section{Ethical standards}

All procedures performed were in accordance with the ethical standards of the institutional and/or national research committee and with the 1975 Helsinki declaration and its later amendments or comparable ethical standards. Local ethics board approval was obtained for this study. Approved by the Ethical Committee on Clinical Research of the Shuyang People's Hospital. Individual patient consent was not required due to nature of study.

\section{Availability of data and materials}

All data generated or analysed during this study are included in this published article.

\section{Authors' contributions}

Dao Ming. Tong conceived and designed the systematic review. Dao Ming. Tong and Ye Ting. Zhou conducted data screening and extraction. All authors participated in the drafting and critical revision of the manuscript. All authors read and approved the final manuscript. 


\section{References}

1. Mosley I, Nicol M, Donnan G, Patrick I, Kerr F, Dewey H. The impact of ambulance practice on acute stroke care. Stroke. 2007;38: 2765-2770.

2. Rosamond WD, Evenson KR, Schroeder EB, Morris DL, Johnson AM, Brice JH. Calling emergency medical services for acute stroke: a study of 9-1-1 tapes. Prehosp Emerg Care. 2005;9: 19-23.

3. Percheron G . Arteries of the human thalamus.II. Arteries and paramedian thalamic territory of the communicating basilar artery. Rev Neurol (Paris),1976,132:309-324.

4. Rivera-Lara L, Henninger N. Delayed sudden coma due to artery of percheron infarction.JAMA Neurol. 2011;68(3):386-7

5. Zappella N, Merceron S, Nifle C, et al. Artery of Percheron infarctionas an unusual cause of coma: three cases and literature review. Neurocrit Care. 2014;20(3):494-501.

6. Turner J, Richardson T, Kane I,et al. Decreased consciousness: bilateral thalamic infarction and its relation to the artery of Percheron.BMJ Case Rep. 2014 16;2014. pii: bcr2013201848.

7. Matheus MG, Castillo M. Imaging of acute bilateral paramedian thalamic and mesencephalic infarcts.AJNR Am J Neuroradiol. 2003;24(10):2005-8.

8. Arauz A, Patiño-Rodríguez HM, Vargas-González JC, et al. Clinical spectrum of artery of Percheron infarct:clinical-radiological correlations. J Stroke Cerebrovasc Dis. 2014;23(5):1083-8.

9. Jumean K, Arqoub AA, Al Hadidi MA, et al .Bilateral thalamic stroke due to occlusion of thearteryof Percheron in a patient with a patent foramen ovale.J Nat Sci Biol Med. 2016;7(1):109-12.

10. Shah AR, Ali R. Artery of Percheron Infarct - a diagnostic and prognostic conundrum! J Pak Med Assoc.2018; 68(1):123-126.

11. Lazzaro NA, Wright B, Castillo $M$, et al. Artery of Percheron infarction: imaging patterns and clinical spectrum. Am J Neuroradiol 2010; 31: 1283 -9.

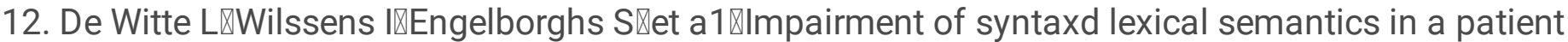
with bilateral paramedian thalamic infarction. Brain Lallg $2006 \otimes 96(1) \varangle 69-77$.

13. Suzuki K, Miyamoto T, Miyamoto M,et al. Hypocretin-1 levels in the cerebrospinal fluid of patients with Percheron artery infarction with or without midbrain involvement: A case series.Medicine (Baltimore). 2016 ;95 (29):e4281.

14. Plun F, Posner JB. The diagnosis of stupor and coma, $3^{\text {rd }}$. ed. Philadelphia: FA Davis Co, 1980

15. Hawkes MA, Arena JE, Rollán C, Pujol-Lereis VA, Romero C, Ameriso SF. Bilateral Paramedian ThalamicInfarction. Neurologist. 2015; 20(5): 89- 92.

16. Cao W, Dong Q, Li L, Dong Y. Bilateral thalamicinfarctionand DSA demonstrated AOP after thrombosis.Acta Radiol Short Rep. 2012; 6;1(1)

17. Usón-Martín M,Gracia-Naya M.Top of the basilar artery syndrome: clinico- radiological aspects of 25 patients. Rev Neurol. 1999; 28(7): 698-701 
18. Delcker A, Diener HC. Neurological diagnosis and therapeutic measures in cerebral embolism. Herz 1991;16:434-43.

19. Hart CL, Hole DJ, Smith GD. The relation between questions indicating transient ischaemic attack and stroke in 20 years of follow up in men and women in the Renfrew/Paisley Study. J Epidemiol Community Health 2001;55:653-6.

20. Collen D. On the regulation and control of fibrinolysis. Edward Kowalski memorial lecture. Thromb Haemost. 1980: 43: 77-79.

21. Lam CK, Yoo T, Hiner B, Liu Z, Grutzendler J. Embolus extravasation is an alternative mechanism for cerebral microvascular recanalization. Nature. 2010;465: 478-482.

22. Liping Liu,Jing Ding ,Xinyi Leng, at el. Guidelines for evaluation and management of cerebral collateral circulation in ischaemic stroke 2017. Stroke and Vescular Neurology. 2017; 56(6):460-471.

23. Gentilini M,De Renzi E,Crisi G. Bilateral paramedian thalamic artery infarcts: report of eight cases.J Neurol Neurosurg Psychiatry. 1987;50(7):900-9.

24. Weidauer $S$, Nichtweiss $M$, Zanella $F E_{\text {,, }}$ et al. Assessment of paramedianthalamic infarcts: MR imaging, clinical features and prognosis. Eur Radiol. 2004;14(9):1615-26.

25. Wenjie Cao®Qiallg Dong \Linxin Li囚et al『Bilateral thalamic infarcction and DSA demonstrated AOP after thrombosis Acta Radiol Short Rep 2012 6ه1(1)『Pii:arsr 2012》

26. Doubal FN, Dennis MS, Wardlaw JM. Characteristics of patients with minor ischaemic strokes and negative MRI: a cross-sectional study. J Neurol Neurosurg Psychiatry. 2011; 82(5): 540-2.

27. Hotter B, Kufner A, Malzahn U, Hohenhaus M, Jungehülsing GJ, Fiebach JB.Validity of negative highresolution diffusion-weighted imaging in transient acute cerebrovascular events.Stroke. 2013;44(9):2598-600.

28. Wang J,Fu X,Jiang C,Liu $\mathrm{H}$, et al .Bilateral paramedian thalamic infarctioninitially presenting as a convulsiveseizure. Case Rep Neurol Med.2013;2013:704952. doi: 10.1155/2013/704952. Epub 2013 May 23.

29. Naganuma M, Hashimoto Y, Matsuura Y, Terasaki T, Hirano T, Uchino M.Two cases of top of the basilar syndrome with onset seizure. Rinsho Shinkeigaku. 2005;45(9):647-51.

30. Xu G, Ma M, Liu X, Hankey GJ. Is there a stroke belt in China and why? Stroke. 2013; 44:1775-1783.

\section{Figures}



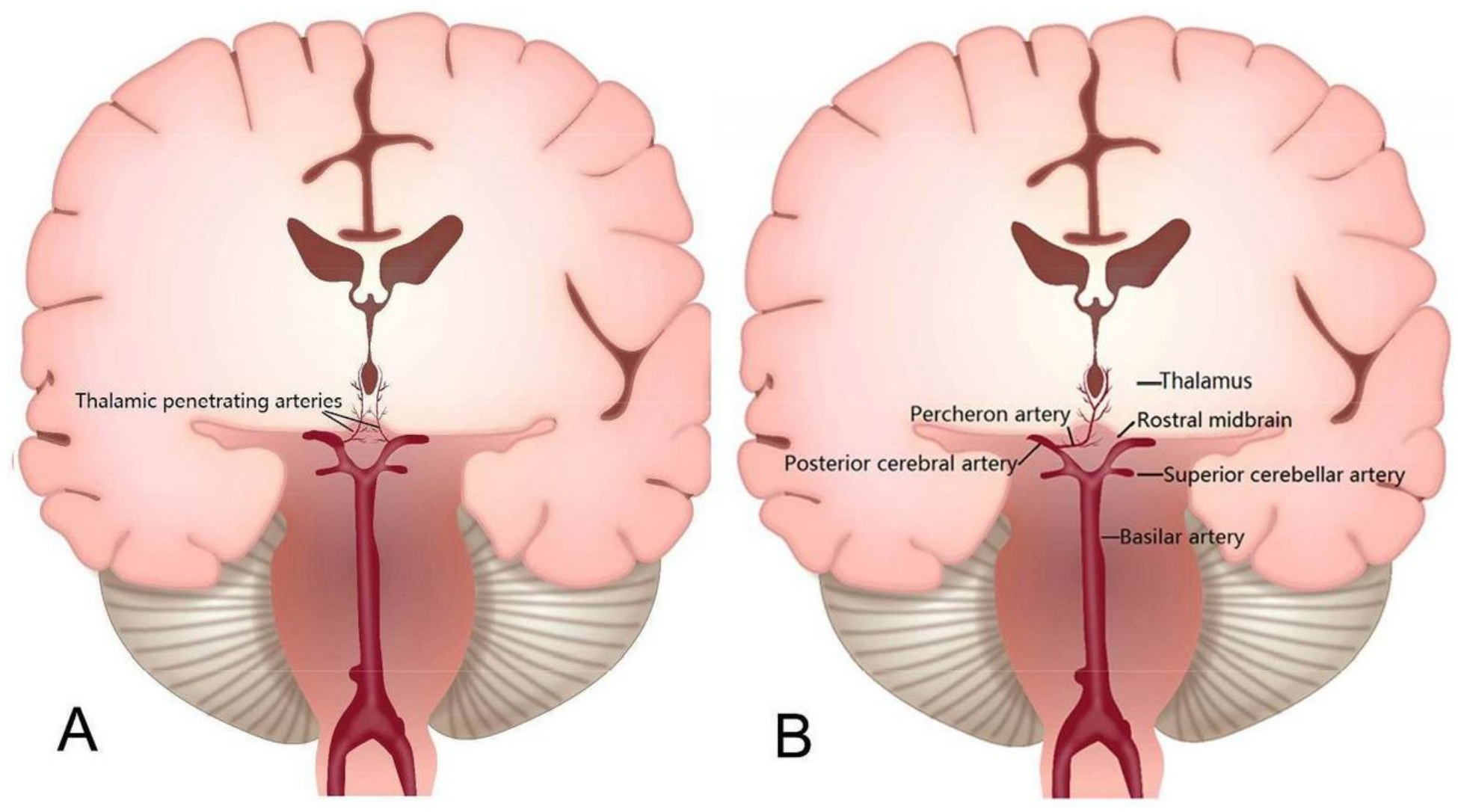

Figure 1

The blood supply of the paramedian thalamus and rostral midbrain. A, Conventional anatomy demonstrating paired thalamic and midbrain perforating arteries. B.The Percheron artery is a solitary arterial trunk that arises from the P1 segment of unilateral posterior cerebral artery and supply blood to the paramedian thalamus and the rostral midbrain bilaterally. 

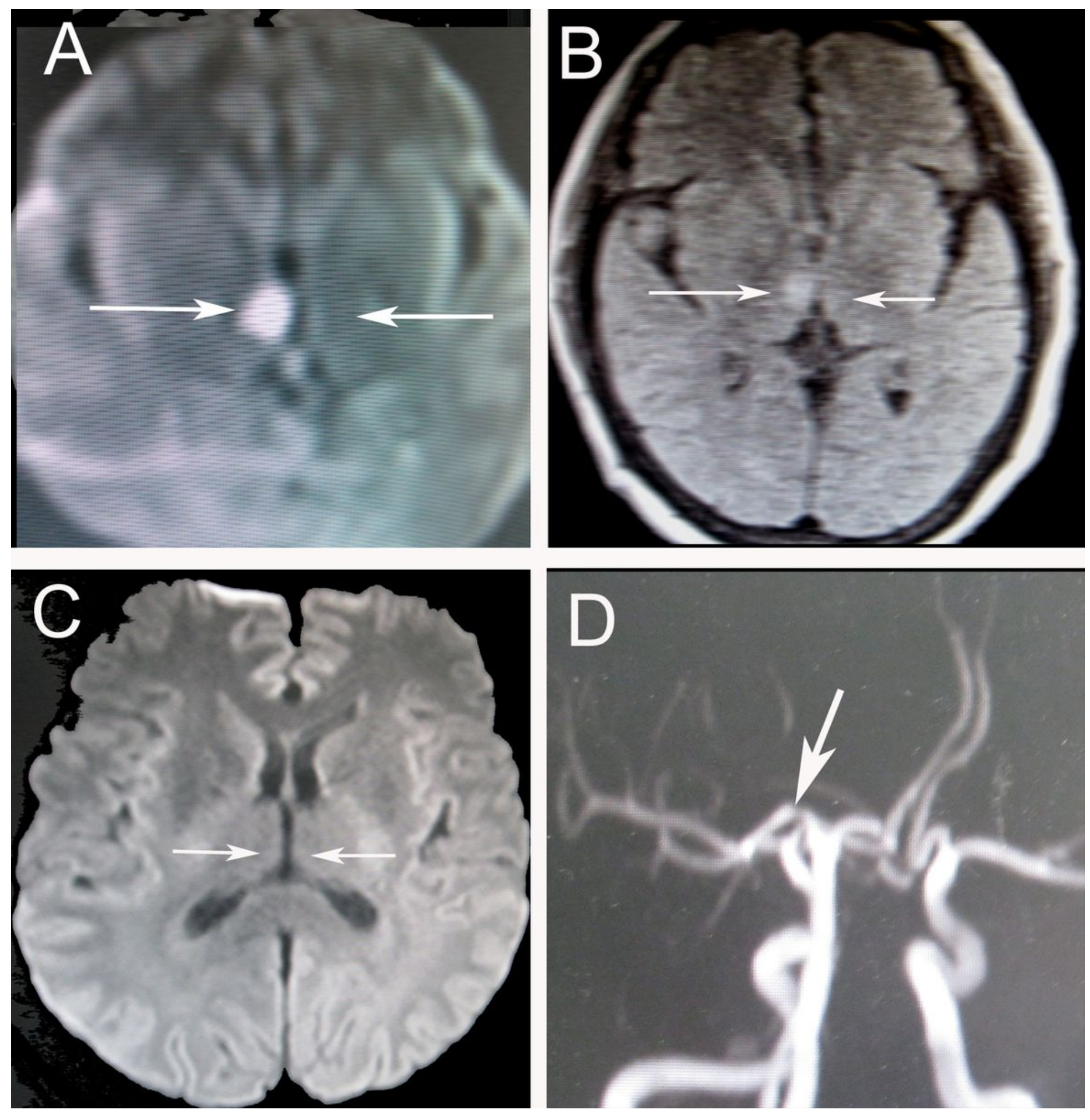

Figure 2

Acute transient PAIC events in the paramedian thalamus/rostral midbrain on brain MRI. Panels A and B show a patient with a 30-min transient coma. At $2 \mathrm{~h}$ after free coma, a new infarction in the right thalamus and a potential natural recanalization of coma event in the left thalamus is verified on the DWI in Panel A (arrow) and on the FLAIR in Panel B (arrow). Panel C shows a patient with a $1.5 \mathrm{~h}$ transient coma. At $3 \mathrm{~h}$ after free coma, DWI shows no any lesions in the bilateral paramedian thalamus, but MRA in 
Panel D (arrow) discloses the stenosis of P1 segment in right posterior cerebral artery, supporting the transient coma with naturally recanalization of infarcts or TIA.

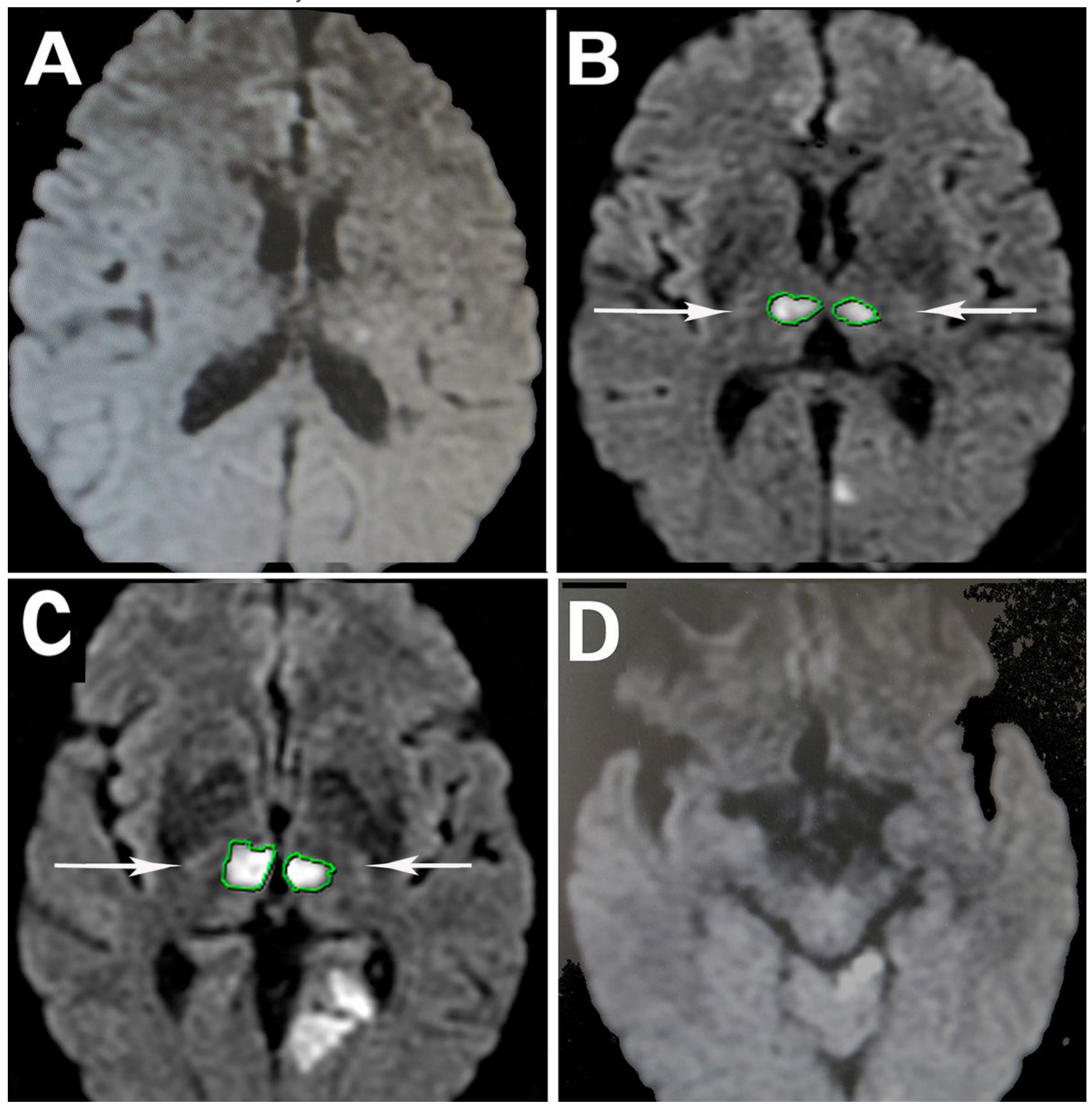

\section{Figure 3}

Irregular shapes infarcts areas on DWI were exactly measured by PACS (per slice). A 62-year-old male who was sudenlly coma $(G C S M<6)$ for $1 \mathrm{~h}$ admited to ICU, his DWI infarct volume $(\mathrm{cm} 3)$ in the paramedian thalamus infarct areas ( all slice in MRI; $1.7 \mathrm{~cm} 3$ ) was calculated by the PACS. He was awaked after persistent coma 22 days. 

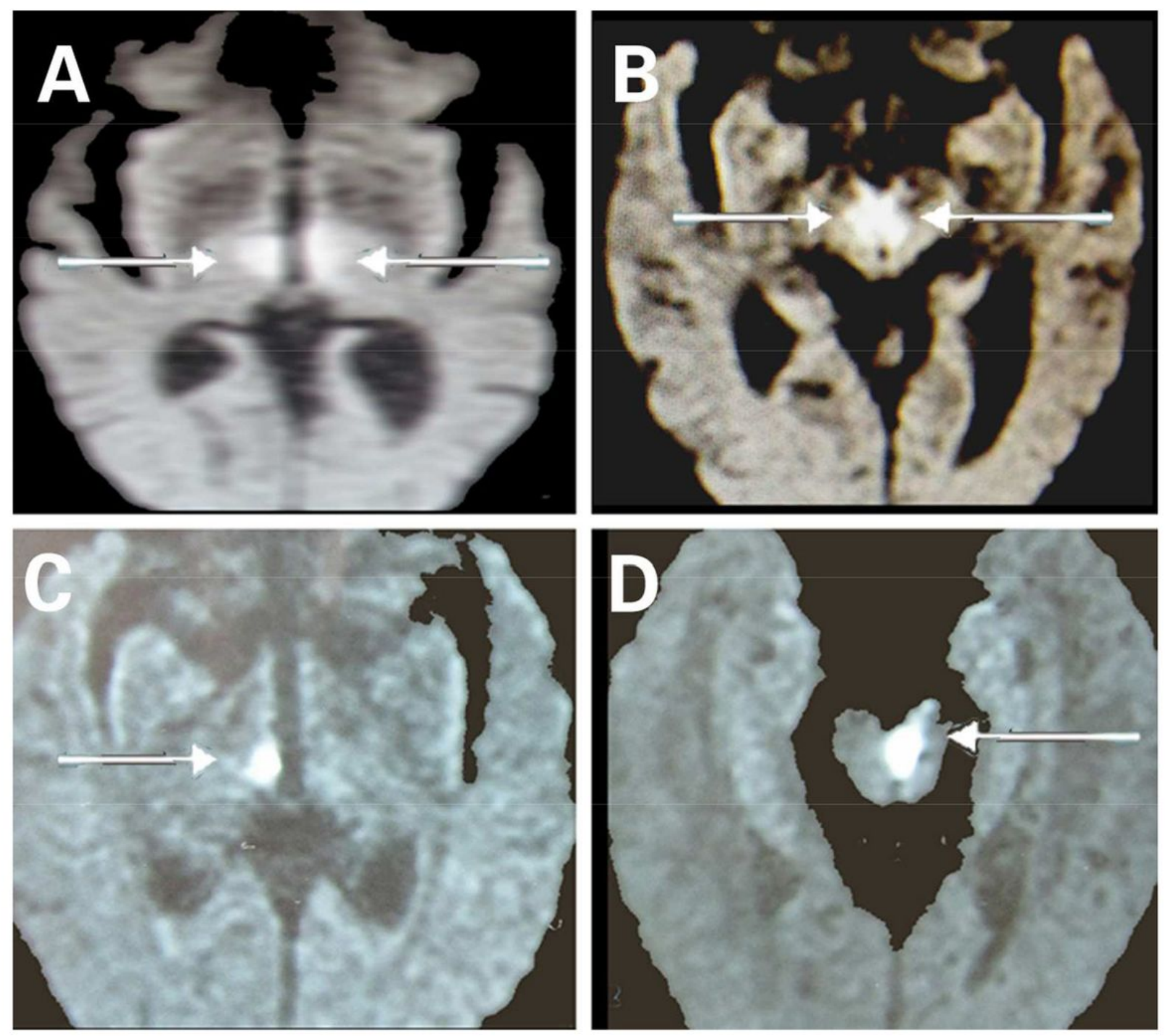

\section{Figure 4}

Acute persistent PAIC with lesions in the bilateral paramedian thalamus/rostral midbrain but no other lesions from the top of basilar artery on DWI. Panels A and B show a 80-year-old male with a persistent PAIC. At $48 \mathrm{~h}$ after persistent coma, new infarction in the bilateral paramedian thalamus and the bilateral midbrain (arrow) are visible on the DWI. Panels $C$ and D show a 55-year-old male with a 36-h persistent PAIC. At $2 \mathrm{~h}$ after free coma, new infarction crossed in the right paramedian thalamus and left rostral midbrain (arrow) are visible on the DWI. 

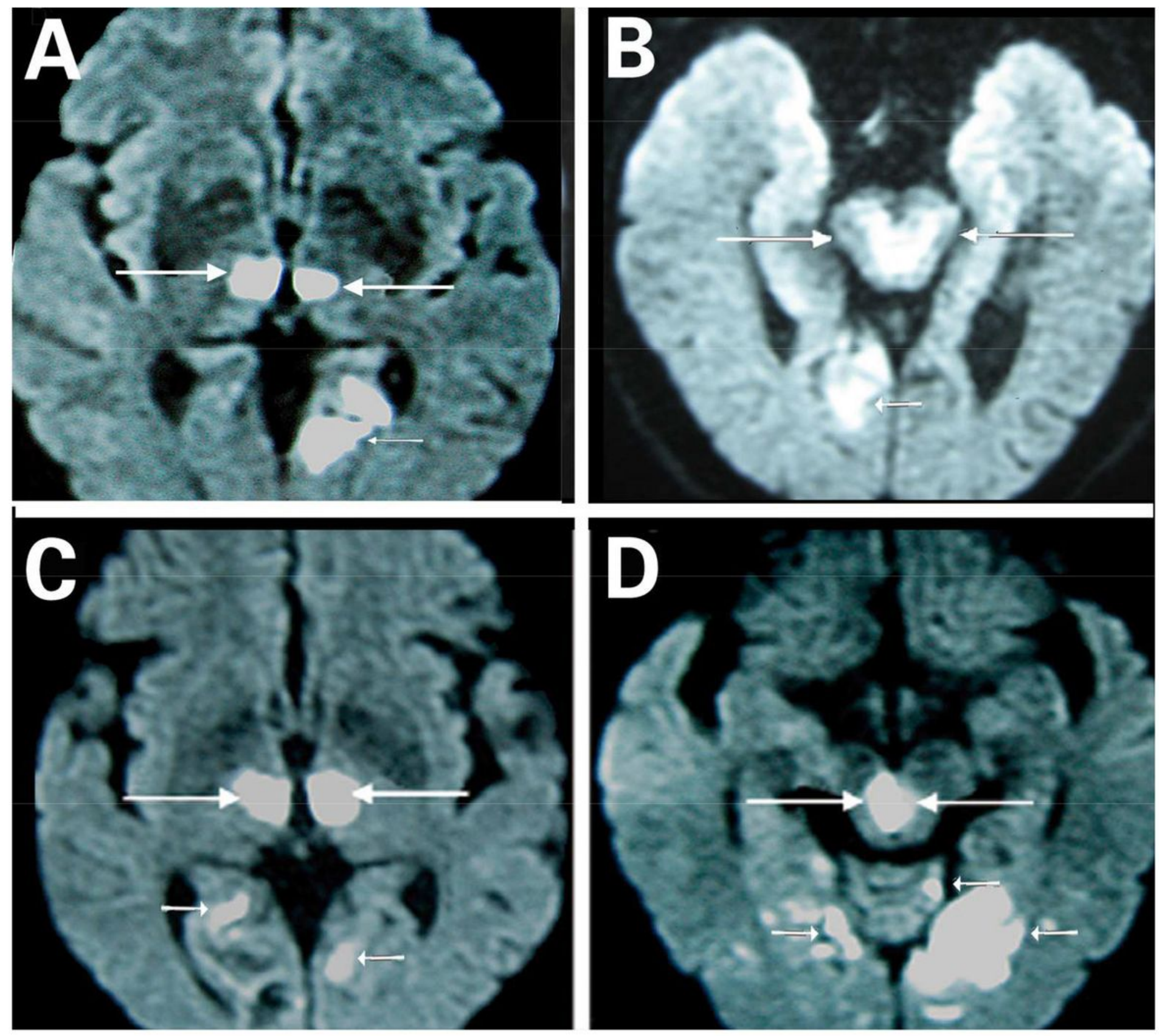

\section{Figure 5}

Acute persistent PAIC with lesiobs in the bilateral paramedian thalamus /rostral midbrain and other lesions from the top of basilar artery on DWI. Panel A and B show a 61-year-old male with sudden persistent PAIC, and the large infarctions in the bilateral paramedian thalamus and rostral midbrain(long arrow) and lesions in the other bencher of the basilar artery (short arrow) are visible on DWI. In panels C and D, DWI shows large infarctions in the bilateral paramedian thalamus and midbrain (long arrow) and lesions in the other branches of the basilar artery (short arrow) in a 74-year-old male with persistent PAIC. 


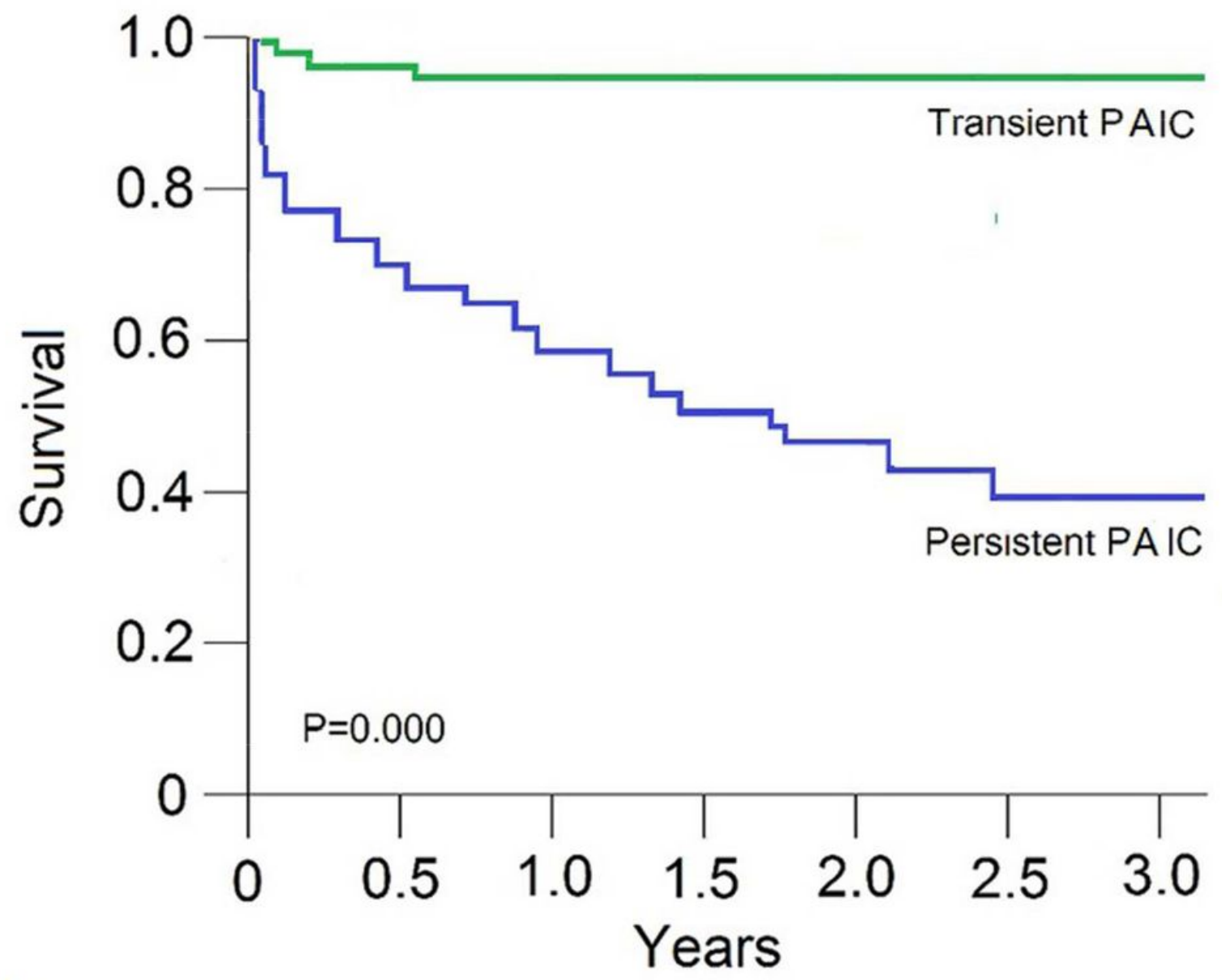

No. at Risk

$\begin{array}{llllllll}\text { Transient PAIC } & 63 & 61 & 60 & 60 & 60 & 60 & 60 \\ \text { Persistent PAIC } & 30 & 18 & 14 & 13 & 13 & 12 & 12\end{array}$

Figure 6

Kaplan-Meier survival curves for patients with PAIC. The Kaplan-Meier survival curves show that patients with persistent PAIC had significantly worse survival than patients with transient PAIC during the 3-year follow-up (hazard ratio $=13.5 ; 95 \% \mathrm{Cl}, 10.29-29.31 ; \mathrm{P}=0.000$ ).

\section{Supplementary Files}

This is a list of supplementary files associated with this preprint. Click to download.

- PAICsupplement.doc 